\title{
Application of Electrostatic Extrusion - Flavour Encapsulation and Controlled Release
}

\author{
Verica Manojlovic ${ }^{1}$, Nevenka Rajic ${ }^{1}$, Jasna Djonlagic ${ }^{1}$, Bojana Obradovic ${ }^{1}$, Viktor Nedovic ${ }^{2}$ \\ and Branko Bugarski ${ }^{1, *}$
}

1 Faculty of Technology and Metallurgy, University of Belgrade, Karnegijeva 4, 11120 Belgrade, Serbia; E-mails: manojlovicverica@yahoo.com (Verica Manojlovic); nena@tmf.bg.ac.yu (Nevenka Rajic); jasna@elab.tmf.bg.ac.yu (Jasna Djonlagic); bojana@tmf.bg.ac.yu (Bojana Obradovic)

2 Department of Food Technology and Biochemistry, Faculty of Agriculture, University in Belgrade, Nemanjina 6, 11081 Belgrade-Zemun, Serbia; E-mail: vnedovic@agrifaculty@bg.ac.yu (Viktor Nedovic)

* Author to whom correspondence should be addressed; E-mail: branko@tmf.bg.ac.yu.

Received: 10 December 2007 / Accepted: 19 February 2008 / Published: 3 March 2008

\begin{abstract}
The subject of this study was the development of flavour alginate formulations aimed for thermally processed foods. Ethyl vanilline was used as the model flavour compound. Electrostatic extrusion was applied for the encapsulation of ethyl vanilline in alginate gel microbeads. The obtained microbeads with approx. $10 \% \mathrm{w} / \mathrm{w}$ of ethyl vanilline encapsulated in about $2 \% \mathrm{w} / \mathrm{w}$ alginate were uniformly sized spheres of about $450 \mu \mathrm{m}$. Chemical characterization by H-NMR spectroscopy revealed that the alginate used in this study had a high content (67\%) of guluronic residues and was rich in GG diad blocks $\left(\mathrm{F}_{\mathrm{GG}}=55 \%\right)$ and thus presented a high-quality immobilisation matrix. The thermal behaviour of alginate beads encapsulating ethyl vanilline was investigated by thermogravimetric (TG) and differential scanning calorimetry measurements (TG-DSC) under heating conditions which mimicked usual food processing to provide information about thermal decomposition of alginate matrix and kinetics of aroma release. Two well resolved weight losses were observed. The first one was in the $50-150{ }^{\circ} \mathrm{C}$ temperature range with the maximum at approx. $112{ }^{\circ} \mathrm{C}$, corresponding to the dehydration of the polymer network. The second loss in the $220-325{ }^{\circ} \mathrm{C}$ temperature range, with a maximum at $\sim 247^{\circ} \mathrm{C}$ corresponded to the release of vanilline. The obtained results indicate that up to $230{ }^{\circ} \mathrm{C}$ most of the vanilline remained intacta, while prolonged heating at elevated temperatures led to the entire loss of the aroma compound.
\end{abstract}


Keywords: electrostatic extrusion, ethyl vanillin, flavour encapsulation, TG-DSC.

\section{Introduction}

Food manufacturers are usually concerned about the preservation of aromatic additives, since aroma compounds are not only delicate and volatile, but also very expensive. Encapsulation provides an effective method to protect flavour compounds from evaporation, degradation, and migration from food [1]. Namely, creating a suitable microenvironment around flavours reduce the volatility and/or mobility of the flavour constituents [2] and provides a better retention during the baking process.

Flavour encapsulation can be accomplished by a variety of methods. The two major industrial processes are spray-drying and extrusion [1]. Encapsulation of flavours via extrusion in glassy carbohydrate matrices has been used for volatile and unstable flavours. The principal advantage of the extrusion method is the stability of the flavour against oxidation. Carbohydrate matrices have very good barrier properties and extrusion is a convenient process enabling the encapsulation of flavours [3]. Hitherto, beads from 200 to 2000 microns have been produced by various extrusion techniques with a maximal flavour load of up to $20 \% \mathrm{w} / \mathrm{w}$ [1].

Recently, a new promising technique for the encapsulation and production of uniform gel microbeads has been developed [4]. The method is based on the use of electrostatic forces to disrupt the liquid filament at the tip of a capillary/needle and to form a charged stream of small droplets. Electrostatic extrusion is a suitable technique for the production of very small particles and has advantages over other extrusion techniques when large-sized capsules negatively affect the textural and sensorial properties of food products. The size of the obtained polymer spheres is a complex function of several operating parameters, the system properties and the properties of the polymer solution [5]. Bugarski et al. [6] preformed excessive investigations to determine the specific influence of each parameter on the diameter of microbeads and the encapsulation efficiency, which led to an optimized method for obtaining particles of uniform and desirable size. This is important for achieving optimal release kinetics, since flavour loss is mass transfer controlled and, accordingly, depends on the bead size.

In this study, calcium alginate gel was employed as the matrix for flavour encapsulation. Alginate has been approved as a coating material by the Food and Drug Administration (US) and European Food Safety Authority (Europe). One of the major advantages of flavour encapsulation in alginate beads is that the encapsulation does not adversely affect the release of the flavour during consumption of the product. The beads provide a sustained release of the flavour to the product during storage and prior to consumption $[7,8]$.

Alginates are naturally derived linear copolymers of 1,4-linked $\beta$-D-mannuronic acid (M) and $\alpha$-L-guluronic acid $(\mathrm{G})$ residues [9-11]. The ratio and sequential distribution of uronic acid residues along the length of the alginate chain vary in alginates of different origins (brown seaweeds, certain bacteria) [9-11]. There is no regular repeat unit in alginate polymers, and the chains can be described as a varying sequence of regions which are usually denoted as M blocks, G blocks, and MG blocks. Aqueous solutions of polysaccharides form hydrogels in the presence of divalent $\mathrm{Ca}^{2+}$ ions via ionic 
interactions between the acid groups on the $\mathrm{G}$ blocks and the gelating ions. As a result, calcium alginate gels are physically cross-linked polymers with mechanical and hosting properties dependant on the alginate composition $[9,10]$.

In this study the electrostatic extrusion technique was applied to immobilize an aromatic compound in calcium alginate gel microbeads. Ethyl vanilline (3-ethoxy-4-hydroxybenzaldehyde) was used as the aroma agent (in the further text vanilline). It is used as a substitute for vanilla (3-methoxy 4-hydroxybenzaldehyde) in foods and perfumes, because it is cheaper and posseses better storage and transport characteristics. Vanilline is an important food additive as a flavour enhancer. This compound is widely used to contribute to the fragrance of commercial foods such as candies, cookies, chocolate and beverages. Although it enhances the scent and taste of food, vanilline must be carefully added. Large amounts of this flavour cause headaches, nausea and vomiting [12]. Since flavour retention and release is influenced by the microstructure of the capsule, the chemical composition of alginate was determined by H-NMR-spectroscopy. In order to determine the smallest amount of the aroma which would give an optimal result, the behavior of the encapsulated aroma under heating conditions which mimic these of usual food processing was investigated by thermoanalytical techniques, TG-DSC analyses. The TG-DSC measurements provide data regarding the melting point, thermal stability, weight loss during heating as well as data, which can served for furter studying of decomposition kinetics. The results could be useful for the formulation studies of food additives as well as for subsequent development of a stable and effective dosage form.

\section{Experimental Section}

\subsection{Materials}

In this study, a low viscosity sodium alginate Protanal LF 20/40, purchased from FMC Biopolymer was used. Vanilline was supplied by Borregaard Synthesis (Sarpsborg, Norway) as a food grade quality (purity $>99.9 \%$ ) and was used without further purification. Calcium chloride dihydrate and potassium sulphate were purchased from SIGMA.

\section{$2.2{ }^{1}$ H NMR spectroscopy}

The mole ratio of mannuronate $(\mathrm{M})$ to guluronate $(\mathrm{G})$ residues $(\mathrm{M} / \mathrm{G})$ and the mole fraction of $\mathrm{GG}$, $\mathrm{MM}$ and $\mathrm{GM}$ diad sequences $\mathrm{F}_{\mathrm{GG}}, \mathrm{F}_{\mathrm{MM}}$ and $\mathrm{F}_{\mathrm{GM}}$ were determined by ${ }^{1} \mathrm{H}$ NMR spectroscopy according to Grasdalen [13]. The alginate sample was partly decomposed under mild hydrolysis in the presence of a dilute acid in order to diminish the viscosity of the solution. The hydrolysed alginate sample was dissolved in $\mathrm{D}_{2} \mathrm{O}$ at neutral $\mathrm{pD}$. The ${ }^{1} \mathrm{H}$ NMR spectra were run at $400 \mathrm{MHz}$ on a Bruker AC $250 \mathrm{E}$ NMR spectrometer.

\subsection{Thermal analysis}

The thermal behavior of the particles was investigated employing the simultaneous TG-DSC technique using a TA Instruments model SDT Q-600 (New Castle, Delaware, US). The samples (mass approx. $20 \mathrm{mg}$ ) were heated in a standard alumina sample pan. All experiments were performed out under dynamic air of a flow rate of $0.1 \mathrm{dm}^{3} / \mathrm{min}$ using a heating rate of $10{ }^{\circ} \mathrm{C} / \mathrm{min}$ per run. 
The experiments were repeated four times to confirm the repeatability and authenticity of the data. The deviations observed are very little.

\subsection{Preparation of samples}

The polymer-vanilline dispersion was prepared by dissolving sodium alginate powder $(0.02 \mathrm{~g} / \mathrm{ml})$ in distilled water containing vanilline $(0.1 \mathrm{~g} / \mathrm{ml})$. Spherical droplets were formed by extrusion of the polymer-vanilline dispersion through a blunt stainless steel needle using a syringe pump (Razel, Scientific Instruments, Stamford, CT) and a $10 \mathrm{ml}$ plastic syringe. The needle was the positively charged electrode, while the collecting solution was grounded. The collecting solution was calcium chloride of $0.015 \mathrm{~g} / \mathrm{ml}$ concentration. The potential difference was controlled by a high voltage dc unit (Model 30R, Bertan Associates, Inc., New York) and was kept at a constant voltage of $4.5 \mathrm{kV}$. The distance between the needle tip (22 gauge) and the collecting solution was $2.5 \mathrm{~cm}$, while the flow rate of the polymer solution was $25.2 \mathrm{~cm}^{3} / \mathrm{h}$. The experimental set-up is shown in Figure 1. A sample of 30 microbeads was taken from each experiment and the diameters of the microbeads were measured with an accuracy of $10 \mu \mathrm{m}$ using a microscope (Carlzeiss Jena). The average microbead diameter and standard deviations were then calculated from the measured data.

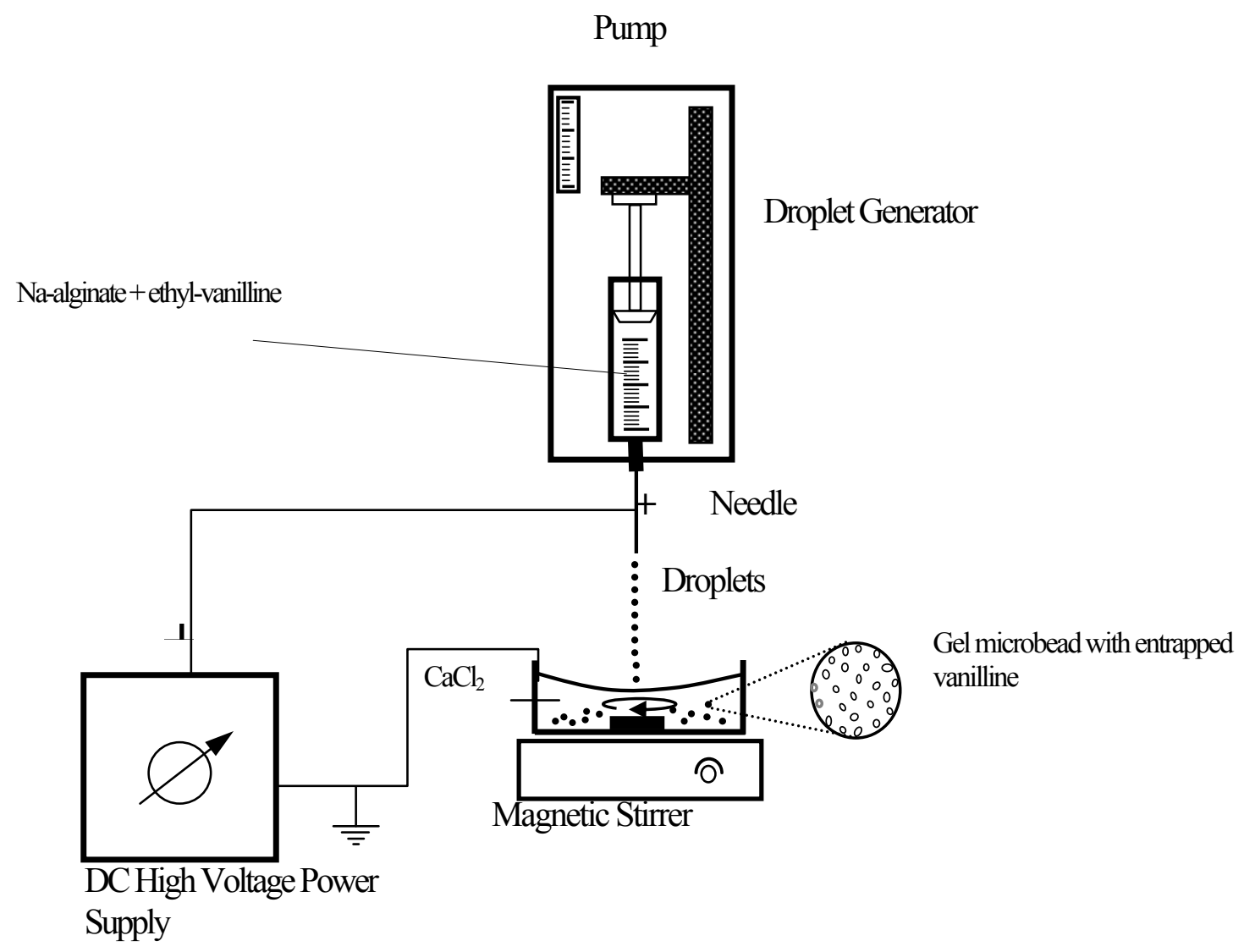

Figure 1. Electrostatic droplet generator. 
Prior to the TG measurements, the alginate microbeads containing vanilline were filtered under a low vacuum and then stored under a solution of saturated potassium sulphate ( $97 \%$ relative humidity) for two hours. It was established that after two hours under conditions of high humidity, the weight loss of the alginate microbeads due to the drying process becomes negligible. It was also found that this step improves the reproducibility of the TG measurements.

\section{Results and Discussion}

The physicochemical and functional properties of alginate molecules within an immobilisation matrix strongly correlate with their composition and block structure. Since the hosting properties of alginate mainly depend on the proportion, as well as the distribution of the two monomer units, the chemical structure of the alginate was determined by H-NMR measurements. The results show that the alginate employed in this study had a high content $(67 \%)$ of guluronic residues and that was rich in GG diad blocks $\left(\mathrm{F}_{\mathrm{GG}}=55 \%\right)$. The mole fractions of the MM and $\mathrm{GM}$ diad sequences, $\mathrm{F}_{\mathrm{MM}}$ and $\mathrm{F}_{\mathrm{GM}}$, were 21 and $12 \%$, respectively. The H-NMR spectra showed no significant peaks originating from proteins or other impurities. They could be bonded to polysaccharide chains of seaweeds from which, alginate is extracted. The chemical composition of the investigated polymer also revealed that it belonged to the group of alginates with a very high content of guluronic residues, classifying it among the high-quality immobilisation matrixes. These matrixes show the presence of open-pore structures and mechanical strength. The gels obtained of alginates with a high G-block content are stronger, more stable and better at retaining incorporated compounds than those of alginates with a low content of G-blocks.

The microbeads incorporating vanilline and produced with the electrostatic droplet generator operating with an applied voltage of $4.5 \mathrm{kV}$ were $450 \pm 20 \mu \mathrm{m}$ in diameter, appearing as regular spheres of a uniform size. Electrostatic extrusion is an effective technique for producing microbeds of desired diameter. By increasing the applied electric potential up to some critical value and adjusting other operating parameters it is possible to reduce particle size, as has been extensively investigated [4]. Compared with empty alginate microbeads produced under same conditions, the obatained particles were slightly larger. It was previously established that incorporation of a material (cells or other active compounds) can cause an increase in the size of the particles [14]. The presence of vanilline molecules in the polymer solution alters the two-phase fluid dynamics in the capillary and the mechanism of droplet formation, which requires additional examination to be fully understood.

\section{Thermal analysis}

The TG and DTG curves of the vanilline encapsulated microbeads in the $20-400{ }^{\circ} \mathrm{C}$ temperature range are shown in Figure 2. Open circles refer to beads weight relative to their initial weight, prior to heating, while black squares represent derivative of relative weight, thus show rate of weight loss. It can be seen that there are two well resolved weight loss ranges. The first weight loss occurs in the 50-150 ${ }^{\circ} \mathrm{C}$ range, with a maximum at approx. $112{ }^{\circ} \mathrm{C}$ on the DTG curve. According to prior measurements of vanilline-free microbeads, this loss can be attributed to the dehydration of the polymer network. This event is also accompanied by an endotherm in the DSC curve (Figure 3). The 
TG curve exhibits a plateau up to $220^{\circ} \mathrm{C}$, indicating that ethyl vanilline remained entrapped inside the polymer matrix. The second weight loss occurs in the $220-325{ }^{\circ} \mathrm{C}$ range and it corresponds to the release of vanilline. There are three DTG maxima in this temperature range and the main one is centred at about $247^{\circ} \mathrm{C}$. The fact that the release of vanilline occurs over a relatively wide range of temperature and that it proceeds in several steps strongly support the conclusion that vanilline is mostly encapsulated inside the polymer matrix (not only physically adsorbed at the surface of the bead) and that the encapsulation enables a slow release of the flavour agent. For a comparison, a cooking process is usually completed below $230{ }^{\circ} \mathrm{C}$. At this temperature most of vanilline remains intacta. TG-DSC measurements of Ca-alginate (the curves are not given) showed that the decomposition of the carrier begins around $155^{\circ} \mathrm{C}$. The two peaks in DTG curves indicate that weight losses occur at around $205{ }^{\circ} \mathrm{C}$ and $300{ }^{\circ} \mathrm{C}$. About $20 \mathrm{wt} . \%$ of the matrix decomposed up to $230{ }^{\circ} \mathrm{C}$, which is usually final backing process temperature .

The analysis showed that all the encapsulated vanilla had been completely released from the capsules during the heating process which can be an undesirable effect when a high retention during the baking processing is required. This is the result of several factors influencing the release of aroma compound from alginate gel microbeads, such as a small size of the capsules (450 microns), the high moisture content ( $\sim 88 \% \mathrm{w} / \mathrm{w})$, and/or absence of oil solvent, which eventually increases the viscosity of the surrounding media and increases the mass transfer resistance. It was found that volatile flavour compounds were encapsulated in oil [15] or propylene glycol [16] environment. In the oil environment, an improved flavour retention was observed. This is a consequence of two factors: the lower volatility of the flavour compounds in a hydrophobic than in a hydrophilic environment, and the increased resistance to mass transfer $[7,15,17]$. As a result, the flavour retained in alginate gel beads during the backing of crackers varied from 20 to $60 \%$ depending on the type of flavour [8]. In a hydrophilic environment, such as that in this study, the rate of vanilline release is more likely controlled by intrinsic mass transfer resistance of the flavour molecules inside the polymer network. Molecular interactions between the polysaccharide and the molecules of the aroma compound are also possible but they need to be examined in further studies.

The retention of flavours encapsulated in alginate beads increases with increasing particle size due to the lower surface area of the particles and the longer distance from the centre of the beads to the surface [15]. However, a compromise regarding size of the capsules has to be achieved concerning two opposite demands: a large size necessary for a prolonged release of the encapsulated flavour, and small-sized beads which are desirable from the viewpoint of textural and sensorial properties of food products. It was found that the retention increases with decreasing moisture content of the beads [15]. The higher the moisture content in the beads is, the higher is the amount of water vapour which develops. The water vapour is the carrier which transfers flavour compounds to the surrounding air, thereby disturbing the phase equilibrium and increasing the driving force for mass transport. The retention in the capsules is also increased with increasing thickness of the capsules and viscosity of the surrounding medium [17]. It is obvious that the heat-stable flavour formulations require extensive research work, including at least, optimization of the alginate concentration, vanilla content, size of microbeads, and heating conditions. 


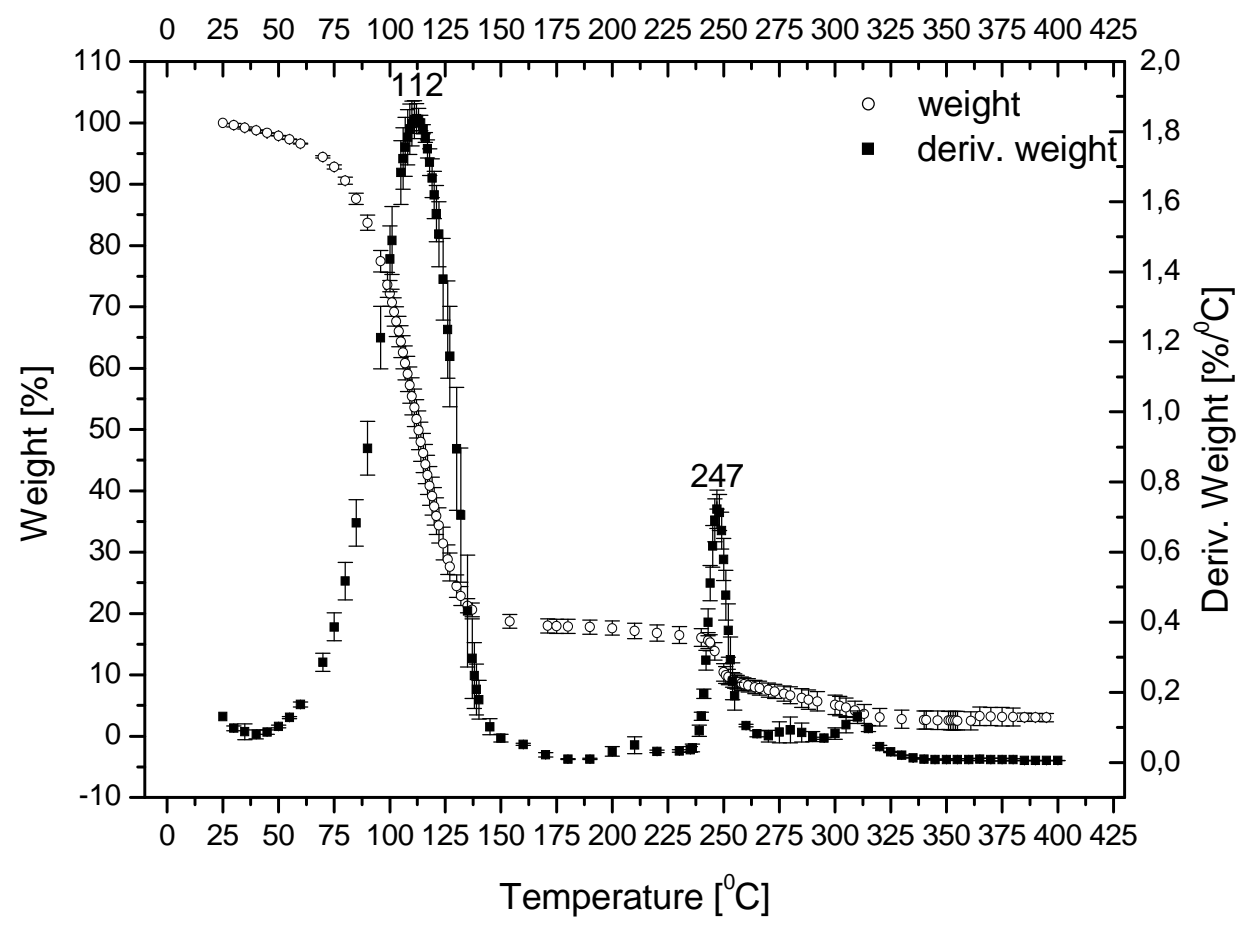

Figure 2. TG and DTG curves of $2 \% \mathrm{w} / \mathrm{w}$ alginate microbeads entrapping approx. $10 \%$ $\mathrm{w} / \mathrm{w}$ ethyl vanilline (data present the average of $n=4$ ).

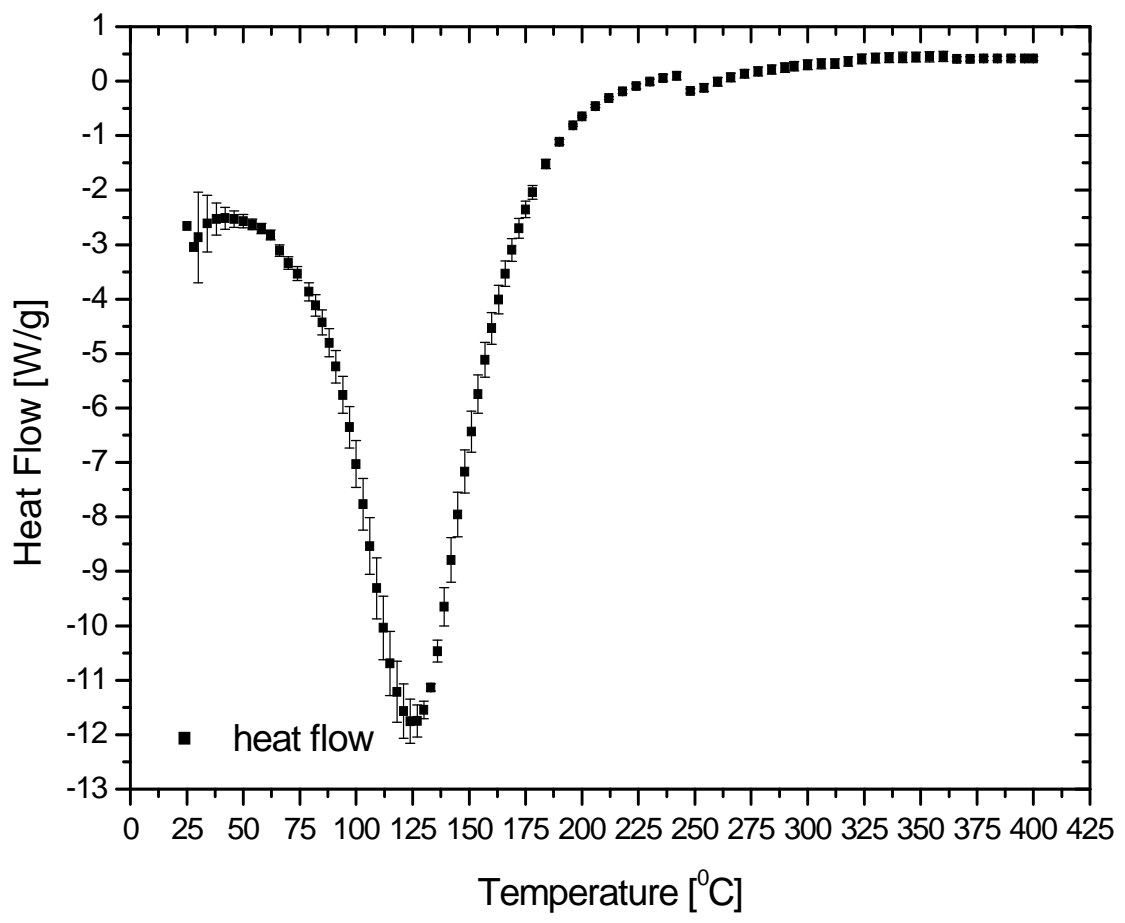

Figure 3. DSC curve of $2 \% \mathrm{w} / \mathrm{w}$ alginate microbeads entrapping approx. $10 \% \mathrm{w} / \mathrm{w}$ ethyl vanilline (data present the average of $n=4$ ). 


\section{Conclusion}

Electrostatic extrusion appears to be a convenient technique for the immobilisation of vanilla into small, monodisperse alginate microbeads. Apparently, TG-DSC is a suitable method for investigating the release of vanilla from alginate microbeads. This study showed that the decomposition process under heating consists of two consecutive, distinctive steps: polymer dehydration and vanilla evaporation. Rupture of weak bonds between alginate chains and water molecules occurs in the $50-150{ }^{\circ} \mathrm{C}$ temperature range and polymer dehydration is most rapid at about $112{ }^{\circ} \mathrm{C}$. Vanilla release begins at a temperature of approx. $225^{\circ} \mathrm{C}$ and rapidly finishes at $247^{\circ} \mathrm{C}$. In order to achieve desirable aroma release, further investigations are planned based on determining the optimal matrix, size of the encapsulating particles and heating conditions. The understanding and control of the complex behaviour of aroma compounds in thermally processed foods require research in both domains: engineering of the matrix with a suitable microstructure and texture properties, as well as the development of the process for the manufacture of microcapsules.

\section{Acknowledgements}

This work was supported by the Ministry of Science, Republic of Serbia.

\section{References and Notes}

1. Madene, A.; Jacquot, M.; Scher, J.; Desorby, S. Flavour encapsulation and controlled release - a review. Int. J. Food Sci. Technol. 2006, 41(1), 1-21.

2. Juteau, A.; Cayot, N.; Chabanet, C.; Doublier, J. L.; Guichard, E. Flavour release from polysaccharide gels: different approaches for the determination of kinetic parameters. Trends Food Sci. Technol. 2004, 15, 394-402.

3. Gouin, S. Microencapulation: industrial appraisal of existing technologies and trends. Trends Food Sci. Technol. 2004, 15, 330-347.

4. Bugarski, B.; Obradovic, B.; Nedovic, V.; Goosen, M. F. A. Electrostatic droplet generation technique for cell immobilization. In Finely dispersed systems; Shu, J. P., Spasic A., Eds.; CRC Press: Boca Raton, FL, 2006; pp. 869-886.

5. Bugarski, B.; Obradovic, B.; Nedovic, V. A.; Poncelet, D. Immobilization of cells and enzymes using an electrostatic droplet generator. In Focus on Biotechnology, Volume 8a: Fundamentals of Cell Immobilisation Biotechnology; Nedovic, V., Willaert, R. G., Eds.; Kluwer Academic Publishers: Dordrecht, 2004; pp. 277-294.

6. Bugarski, B.; Li, Q.; Goosen, M.F.A.; Poncelet, D.; Neufeld, R.J.; Vunjak, G. Electrostatic droplet generation: mechanism of polymer droplet formation. AlChE J. 1994, 40(6), 1026-1032.

7. De Roos, K.B. Understanding and controlling the behavior of aroma compounds in thermally processed foods. Trends Food Sci. Technol. 2006, 17(5), 236-243.

8. De Roos, K.B. Effect of texture and microstructure on flavour retention and release. Int. Dairy J. 2003, 13, 593-605. 
9. Martinsen, A.; Skjak-Braek, G.; Smidsrod, O. Alginate as Immobilization Material; I. Correlation between Chemical and Physical Properties of Alginate Gel Beads. Biotechnol. Bioeng. 1989, 33, 79-89.

10. Gombotz, W.R.; Wee, S.F. Protein release from alginate matrices. Adv. Drug Deliv. Rev. 1998, 31, 267-285.

11. Moe, S.T.; Draget, K.I.; Skjak-Braek, G.; Smidsrod, O. Alginates. In Food Polysaccharides and Their Applications; Stephen A. M., Ed.; Marcel Dekker: New York, 1995; pp. 245-286.

12. Ni, Y.; Zhang, G.; Kokot, S. Simultaneous spectrophotometric determination of maltol, vanilline, and ethyl vanilline in foods by multivariate calibration and artificial neural network. Food Chem. 2005, 89, 465-473.

13. Grasdalen, H.; Larsen, B.; Smidsrod O. Study of the Composition of Uronate Residues in Alginates. Carbohydr. Res. 1979, 68, 23-31.

14. Manojlovic, V.; Djonlagic, J.; Obradovic, B.; Nedovic, V.; Bugarski, B. Investigations of cell immobilization in alginate: rheological and electrostatic extrusion studies. J. Chem. Technol. Biotechnol. 2006, 81, 505-510.

15. Bouwmeesters, J. F.; De Roos, K. B. Process for preparing beads as food additives, (1998) WO 98/15192.

16. Chattopadhyaya, S.; Sindghal, R.S.; Kulkarni, P.R. Oxidised starch as a gum Arabic substitute for encapsulation of flavours. Carbohydr. Polym. 1998, 37, 143-144.

17. Terta, M.; Georgios, B.; Paraskevopoulou, A. Retention of selected aroma compounds by polysaccharide solutions: A thermodynamic and kinetic approach. Food Hydrocol. 2006, 20(6), 863-871.

(C) 2008 by MDPI (http://www.mdpi.org). Reproduction is permitted for noncommercial purposes. 